\title{
Các yếu tố ảnh hưởng đến đổi mới công nghệ tại các doanh nghiệp vừa và nhỏ của Việt Nam
}

\section{Factors affect the technology innovation at small and medium Vietnamese enterprises}

\author{
Nguyễn Thị Anh Vân ${ }^{1 *}$, Nguyễn Khắc Hiếu ${ }^{1}$ \\ ${ }^{1}$ Khoa Kinh Tế- ĐH Sư Phạm Kỹ Thuật TPHCM, Việt Nam \\ *Tác giả liên hệ, Email: anhvan@ @cmute.edu.vn
}

THÔNG TIN

DOI: $10.46223 /$ HCMCOUJS. econ.vi.15.3.1339.2020

Ngày nhận: 24/09/2019

Ngày nhận lại: 16/03/2020

Duyệt đăng: 07/05/2020

Tù khóa:

đổi mới công nghệ, doanh nghiệp vừa và nhỏ, hồi quy Logit

Keywords:

technology innovation, Small and Medium Enterprises (SMEs), Logit regression

\section{TÓM TÁT}

Bài viết nghiên cứu những yếu tố ảnh hưởng đến khả năng đổi mới công nghệ tại các doanh nghiệp vừa và nhỏ (DNVVN) của Việt Nam. Nghiên cứu sử dụng phương pháp hồi quy logit với dữ liệu thời điểm gồm 2649 doanh nghiệp vừa và nhỏ của Việt Nam. Kết quả nghiên cứu cho thấy, có các yếu tố quy mô doanh nghiệp, sự kiểm tra của cơ quan chức năng, chứng nhận chất lượng quốc tế, xuất khẩu, đổi mới sản phẩm và hỗ trợ kỹ thuật từ chính phủ ảnh hưởng tích cực đến đến việc đổi mới công nghệ, trong khi đó thì yếu tố chi phí phi chính thức ảnh hưởng tiêu cực đến việc đổi mới công nghệ. Từ kết quả nghiên cứu, một số kiến nghị được đưa ra nhằm gia tăng việc đổi mới công nghệ tại các doanh nghiệp vừa và nhỏ của Việt Nam.

ABSTRACT
This research focuses on factors affectting technology
innovation at small and medium Vietnamese enterprises (SMEs).
Logit regression is used with crossectional data of 2469 small and
medium Vietnammese enterprises. The results show that some
factors have positive effect on technology innovation such as firm
size, technical compliance inspections by government officials,
international quality certification, exports, product innovation, and
technical assistance of Government whereas informal cost has
negative impact on technology innovation. From the results, some
solutions have been suggested in order to increase technology
innovation of small and medium enterprises in Vietnam.

\section{Giới thiệu}

Hiện nay, với cuộc cách mạng 4.0, công nghệ đang là mấu chốt để quyết định thành bại của nhiều doanh nghiệp. Trên thế giới, việc đổi mới công nghệ diễn ra một cách nhanh chóng và mạnh mẽ. Tuy nhiên ở Việt Nam, đặc biệt tại các doanh nghiệp vừa và nhỏ, vấn đề đổi mới công nghệ đang là một thách thức. Theo thống kê của Bộ Khoa học và Công nghệ (KH và $\mathrm{CN})$ cho thấy, năm 2015 cả nước có gần 600 nghìn doanh nghiệp, với hơn $90 \%$ là DNVVN và phần lớn các doanh nghiệp này đều đang sử dụng công nghệ tụt hậu so với mức trung bình của thế giới từ hai đến ba thế hệ (Minh Nhat, 2015). Và theo báo cáo Năng lực cạnh tranh toàn cầu năm 
2019 của Diễn đàn kinh tế thế giới (WEF) cho thấy, trình độ công nghệ của Việt Nam hiện nay đang xếp hạng 67/141 quốc gia được khảo sát. Trong khi đó việc đổi mới công nghệ có tương quan thuận đến tăng trưởng của doanh nghiệp, tức là việc đổi mới công nghệ có khả năng tăng doanh thu, lợi nhuận, và khả năng cạnh tranh cho doanh nghiệp (UNITED NATIONS UNIVERSITY, 2015). Theo khảo sát các DNVVN cho thấy năm 2015 chỉ 4,9\% các DN được khảo sát có đổi mới công nghệ (UNITED NATIONS UNIVERSITY, 2015). Tại sao tỷ lệ các doanh nghiệp vừa và nhỏ của Việt Nam đổi mới công nghệ thấp? Các yếu tố nào ảnh hưởng đến việc đổi mới công nghệ của các doanh nghiệp vừa và nhỏ tại Việt Nam? Đây là những câu hỏi nghiên cứu quan trọng cần được trả lời. Tuy nhiên, các nghiên cứu về đổi mới công nghệ tại Việt Nam hầu hết chỉ mang tính thống kê và các nhận định của các chuyên gia (Kim Hien, 2015; Minh Nhat, 2015; Nguyen, $\mathrm{Vu}$, \& Nguyen 2018), rất ít nghiên cứu sử dụng phương pháp định lượng để xác định các yếu tố ảnh hưởng đến việc đổi mới công nghệ tại DNVVN.

Chính vì vậy tác giả thực hiện nghiên cứu "Các yếu tố ảnh hưởng đến đổi mới công nghệ tại các công ty vừa và nhỏ của Việt Nam”. Nghiên cứu nhằm xác định các yếu tố ảnh hưởng đến việc đổi mới công nghệ tại các DNVVN của Việt Nam, từ đó đưa ra một số kiến nghị nhằm gia tăng hoạt động đổi mới công nghệ tại Việt Nam.

\section{Cơ sở lý thuyết}

\subsection{Các khái niệm liên quan}

\subsubsection{Khái niệm liên quan đến đổi mới công nghệ}

\section{Đổi mới công nghệ:}

Theo OECD (2005), đổi mới công nghệ bao gồm các sản phẩm và quy trình mới và những thay đổi công nghệ quan trọng của sản phẩm và quy trình. Một đổi mới đã được thực hiện nếu nó đã được giới thiệu trên thị trường. Đổi mới công nghệ là không thể tránh khỏi đối với các công ty muốn phát triển và duy trì tính cạnh tranh cạnh hoặc đạt được mục tiêu thâm nhập vào thị trường mới (Souitaris, 2002). Đổi mới công nghệ là việc thay thế một phần chính hay toàn bộ công nghệ đang sử dụng bằng một công nghệ khác tiến tiến hơn, hiệu quả hơn. Đổi mới công nghệ có thể nhằm tăng năng suất, chất lượng, hiệu quả của quá trình sản xuất hoặc có thể nhằm tạo ra một sản phẩm, dịch vụ mới phục vụ thị trường.

Theo Branscomb (2001), đổi mới công nghệ là việc thực hiện thành công (trong thương mại hoặc quản lý) của một ý tưởng kỹ thuật mới. Những đổi mới được phân biệt với các phát minh. Theo Frankelius (2009) đổi mới có liên quan đến, nhưng không giống như phát minh, vì đổi mới có nhiều khả năng liên quan đến việc triển khai thực tế một phát minh (tức là khả năng mới / cải tiến) để tạo ra tác động có ý nghĩa trong thị trường hoặc xã hội, và không phải tất cả các đổi mới đòi hỏi một phát minh (Bhasin, 2012).

Đổi mới công nghệ đóng một vai trò ngày càng nổi bật trong sự tăng trưởng của các nền kinh tế công nghiệp hàng đầu. Các mô hình của quá trình đổi mới công nghệ đã phát triển theo thời gian và hiện có thể tính đến nhiều yếu tố bên ngoài công ty ảnh hưởng đến khả năng đổi mới (Branscomb, 2001).

Theo Cancino, Paza, Ramaprasad, và Syn (2018), đổi mới công nghệ được coi là phương tiện để tối ưu hóa việc sử dụng hiệu quả các nguồn lực quan trọng trong các hệ thống kinh tế xã hội-sinh học.

Trong nghiên cứu này, nhóm tác giả sử dụng khái niệm đổi mới công nghệ là sự thay đổi trong quy trình hoặc trong công nghệ của các máy móc thiết bị. 


\subsubsection{Doanh nghiệp vìa và nhỏ (DNVVN)}

Theo nghị định số 56/2009/NĐ-CP của chính phủ, nếu doanh nghiệp có từ trên 10 đến 200 lao động là doanh nghiệp có quy mô nhỏ và doanh nghiệp có số lao động từ trên 200 đến 300 là doanh nghiệp có quy mô vừa. Định nghĩa trên áp dụng cho tất cả các ngành trừ thương mại và dịch vụ. Đối với ngành thương mại và dịch vụ, doanh nghiệp nhỏ có số lao động từ trên 10 đến 50 , doanh nghiệp vừa có số lao động từ trên 50 đến 100 . Trong nghiên cứu này khái niệm doanh nghiệp nhỏ và vừa được hiểu theo định nghĩa trên.

\subsection{Các nghiên cúu thục nghiệm liên quan}

Hiện nay, vấn đề đổi mới công nghệ đang được quan tâm mạnh mẽ trên thế giới, đặc biệt với sự phát triển mạng mẽ của cuộc cách mạng công nghiệp 4.0. Vì vậy có khá nhiều nghiên cứu liên quan đến lĩnh vực này. Các nghiên cứu tập trung về hai khía cạnh: các động cơ của để các công ty đổi mới công nghệ và ảnh hưởng của việc đổi mới công nghệ đến công ty như doanh thu, lợi nhuận, chi phí, tính cạnh tranh.... Trong phạm vi liên quan đến đề tài, tác giả lược khảo một số nghiên cứu liên quan đến câu hỏi các yếu tố nào ảnh hướng đến việc đổi mới công nghệ trong công ty. Theo Gnyawali và Park (2009) cho rằng các doanh nghiệp vừa và nhỏ đối mặt với những thách thức to lớn khi cố gắng theo đuổi các đổi mới công nghệ. Chính vì vậy họ đã đưa ra một mô hình khái niệm gồm nhiều yếu tố để kích thích sự áp dụng đổi mới công nghệ cho các nhà quản lý DNVVN và các nhà hoạch định chính sách. Mô hình chỉ ra rằng sự hợp tác chiến lược tức là vừa hợp tác vừa cạnh tranh sẽ giúp các doanh nghiệp vừa và nhỏ có khả năng đổi mới công nghệ. Trong đó chiến lược hợp tác ngành bao gồm: vòng đời sản phẩm ngắn; sự hội tụ công nghệ, chi phí nghiên cứu và phát triển cao.

Tiếp theo Uzkurt, Kumar, Kimzan, và Sert (2012) nghiên cứu ảnh hưởng của sự biến động của môi trường đến sự đổi mới tại doanh nghiệp. Nhóm nghiên cứu khảo sát 156 doanh nghiệp DNVVN tại Thổ Nhỉ Kỳ. Kết quả cho thấy sự biến động về thị trường và sự biến động về công nghệ có ảnh hưởng tích cực đến đổi mới tại các doanh nghiệp DNVVN. Từ kết quả nghiên cứu một số gợi ý được đưa ra nhằm tăng cường hoạt động đổi mới tại doanh nghiệp.

Tiếp theo là một nghiên cứu tại khu vực Đông Nam Á, Thong (2015) nghiên cứu một mô hình về đổi mới công nghệ ở các doanh nghiệp nhỏ ở Singapore. Cuộc điều tra thông qua bảng câu hỏi được tiến hành tại 166 doanh nghiệp, đối tượng trả lời là các CEO của công ty. Kết quả phân tích dữ liệu cho thấy đặc điểm tính cách của CEO (tính sáng tạo, kiến thức về đổi mới); đặc điểm của đổi mới và đặc điểm của tổ chức (quy mô doanh nghiệp và mức độ hiểu biết của nhân viên) ảnh hưởng đến khả năng chấp nhận công nghệ. Ngược lại, đặc tính môi trường cạnh tranh không ảnh hưởng trực tiếp đến việc chấp nhận đổi mới công nghệ của các doanh nghiệp nhỏ.

Gần đây, Rangus và Slavec (2017) nghiên cứu xem xét việc phân quyền, khả năng hấp thu và sự tham gia của nhân viên (dựa trên sáng kiến đổi mới) tác động đến sự đổi mới của công ty. Nghiên cứu khảo sát 421 doanh nghiệp tại sản xuất và dịch vụ tại Slovenia. Kết quả nghiên cứu cho thấy việc phân quyền có ảnh hưởng tích cực đến việc đổi mới tại doanh nghiệp. Ngoài ra việc đổi mới cũng ảnh hưởng tích cực đến kết quả hoạt động của doanh nghiệp.

Tại Việt Nam, trong cuộc cách mạng công nghiệp 4.0 và sự toàn cầu hóa mạnh mẽ như hiện nay thì việc đổi mới công nghệ thực sự đang là vấn đề cấp bách đối với các doanh nghiệp vừa và nhỏ. Tuy nhiên theo nhận định của các chuyên gia thì tỷ lệ với các doanh nghiệp vừa và nhỏ Việt Nam đổi mới công nghệ rất thấp. Chính vì vậy có khá nhiều đề tài phân tích về các nguyên nhân của việc hạn chế đổi mới công nghệ tại DNVVN. Ví dụ như Kim Hien (2015) cho rằng sử dụng công nghệ lạc hậu, phần lớn các doanh nghiệp của nước ta mới chỉ tham gia vào khâu thấp nhất trong chuỗi cung ứng là lắp ráp và gia công, cung cấp các phụ tùng thay thế, chứ chưa tham 
gia sản xuất các sản phẩm có giá trị gia tăng cao. Lý giải về việc này, Viện Nghiên cứu Quản lý Kinh tế Trung ương công bố ngày 26/05/2015 trong báo cáo điều tra Năng lực cạnh tranh và công nghiệp ở cấp độ doanh nghiệp tại Việt Nam cho thấy, trở ngại lớn nhất của doanh nghiệp phải đối mặt chính là trở ngại chính. Theo đó, có tới $90 \%$ trong tổng số 8.000 doanh nghiệp được điều tra (trong thời gian 4 năm) cho biết do gặp khó khăn về tài chính nên họ chưa có chiến lược cải tiến công nghệ. Báo cáo điều tra cũng cho biết, một trong những lý do gây cản trở doanh nghiệp đầu tư công nghệ là do môi trường kinh doanh và điều kiện sản xuất kinh doanh của doanh nghiệp vẫn ở trong tình trạng khó khăn. "Phần lớn các doanh nghiệp Việt Nam dựa vào vốn chủ sở hữu để cải tiến công nghệ, điều đó cho thấy khả năng đầu tư của doanh nghiệp bị hạn chế nguồn vốn có sẵn, ví dụ như lợi nhuận giữ lại”, báo cáo nêu rõ.

Theo Minh Nhat (2015) trích dẫn từ thống kê của Bộ Khoa học và Công nghệ (KH và CN) cho thấy "hiện nay cả nước có gần 600.000 doanh nghiệp, với hơn $90 \%$ là DNVVN. Phần lớn đều đang sử dụng công nghệ tụt hậu so với mức trung bình của thế giới từ hai đến ba thế hệ. Trong đó có đến $76 \%$ máy móc, dây chuyền công nghệ nhập thuộc thế hệ những năm 60 - 70 của thế kỷ trước; $75 \%$ số thiết bị đã hết khấu hao; $50 \%$ thiết bị là đồ tân trang...; chỉ có $20 \%$ là nhóm ngành sử dụng công nghệ cao".

Gần đây, theo Nguyen và cộng sự (2018), DNVVN phải được coi là trung tâm của quá trình đổi mới công nghệ tại Việt Nam vì DNVVN chiếm khoảng $97 \%$ tổng số doanh nghiệp của cả nước, đóng vai trò quan trọng trong việc tạo việc làm, cải thiện thu nhập cho người lao động và huy động nguồn lực cho phát triển kinh tế. Tuy nhiên các quỹ nhà nước hỗ trợ đổi mới công nghệ cho các DNVVN chưa phổ biến ở các quốc gia đang phát triển như Việt Nam.

Tóm lại, các nghiên cứu về đổi mới công nghệ tại Việt Nam hầu hết chỉ mang tính thống kê và các nhận định của các chuyên gia, rất ít nghiên cứu sử dụng phương pháp định lượng để xác định các yếu tố ảnh hưởng đến việc đổi mới công nghệ tại DNVVN. Đề tài này sử dụng phương pháp định lượng nhằm xác định các yếu tố ảnh hưởng đến việc đổi mới công nghệ tại $\mathrm{DNVVN}$ thông qua phân tích dữ liệu 2649 DNVVN tại Việt Nam để các nhà nghiên cứu có cái nhìn khách quan và cụ thể hơn về vấn đề này.

Sau khi lược khảo các nghiên cứu trước, tác giả đề xuất đưa vào mô hình các yếu tố ảnh hưởng đến việc đổi mới công nghệ của các doanh nghiệp vừa và nhỏ như sau:

Đặc điểm chủ sở hũu: Theo Thong (2015) đặc điểm tính cách của CEO (tính sáng tạo, kiến thức về đổi mới) ảnh hướng tích cực đến đổi mới công nghệ trong khi đó tại các công ty vừa và nhỏ thì $\mathrm{CEO}$ thường là chủ sở hữu. Theo Divisekera và Nguyen (2018), đặc điểm chủ sở hữu là yếu tố ảnh hưởng quan trọng đến việc đổi mới trong doanh nghiệp. Sarooghi, Libaers, và Burkemper (2015) cũng cho rằng sự sáng tạo của cá nhân trong tổ chức sẽ ảnh hưởng thuận đến việc đổi mới. Trong một doanh nghiệp nhỏ, chủ sở hữu thường chính là người ra quyết định, đặc điểm của chủ sở hữu là rất quan trọng trong việc xác định thái độ đổi mới của doanh nghiệp. Điều này là do phẩm chất của chủ sở hữu là các yếu tố quyết định phong cách quản lý chung của doanh nghiệp. Chính vì vậy, trong nghiên cứu này, tác giả đưa đặc điểm chủ sở hữu làm biến độc lập với các quan sát sau: giới tính, trình độ học vấn, mức độ chấp nhận rủi ro và ý tưởng đổi mới sáng tạo. Từ đó nhóm tác giả đưa ra giả thuyết:

H1: Đặc điểm chủ sở hũu có ảnh hwởng đến việc đổi mới công nghệ tron doanh nghiệp, cu thể: Nếu trình độ hoc vấn chủ sở hũu càng cao thì khả năng áp dụng đổi mới công nghệ càng cao, Chủ sở hũu có nhiều ý tương đổi mới thì khả năng áp dụng đổi mó́i công nghệ càng cao và chủ sở hũu thích rủi ro thì khả năng áp dụng đổi mới công nghệ càng cao

Quy mô doanh nghiệp: Nhiều nghiên cứu đã khẳng định tác động của quy mô doanh 
nghiệp đối với đổi mới sáng tạo. Theo Raisch và Birkinshaw (2008), các công ty lớn thường có các nguồn lực để thực hiện hiệu quả các hoạt động liên quan đến việc tạo ý tưởng và thực hiện ý tưởng, trong khi các doanh nghiệp nhỏ ít tài nguyên phải đưa ra lựa chọn và có thể không theo đuổi chiến lược để khiến công ty có thể đổi mới. Bên cạnh đó, các công ty lớn hơn có tài chính, tiếp thị tốt hơn, khả năng nghiên cứu mạnh mẽ hơn và kinh nghiệm phát triển sản phẩm / quy trình sâu hơn sẽ tạo điều kiện cho việc chuyển đổi ý tưởng sáng tạo vào các sản phẩm và quy trình mới (Azadegan, Patel, \& Parida, 2013; Branzei \& Vertinsky, 2006). Các nghiên cứu khác đã lập luận rằng các công ty nhỏ hơn gặp bất lợi so với các công ty lớn hơn vì họ không có hệ thống hành chính phân cấp để giúp quản lý các quá trình kiến thức, hạn chế mức độ đổi mới mà họ có thể đạt được (Lubatkin, Simsek, Ling, \& Veiga, 2006). Từ các nghiên cứu trước, ta thấy quy mô doanh nghiệp có vai trò quan trọng đến đổi mới. Trong nghiên cứu này, quy mô doanh nghiệp được đại diện bởi các biến: chi phí nghiên cứu và phát triển, doanh thu và số lượng lao động của doanh nghiệp. Từ đó nhóm tác giả đưa ra giả thuyết:

\section{H2: Doanh nghiệp có quy mô càng lớn thì khả năng áp dụng đổi mới công nghệ càng cao}

Múc độ cạnh tranh: Theo Divisekera và Nguyen (2018) nghiên cứu sự đổi mới trong ngành du lịch tại Australia đã kết luận đặc điểm chủ sở hữu, cạnh tranh, quy mô công ty và môi trường là các yếu tố ảnh hướng đến đổi mới. Mức độ cạnh tranh càng cao thì khả năng doanh nghiệp cần đổi mới công nghệ càng lớn. Chính vì vậy trong nghiên cứu này, tác giả đưa biến mức độ cạnh tranh vào mô hình làm biến độc lập. Từ đó nhóm tác giả đưa ra giả thuyết:

H3: Doanh nghiệp có mức độ cạnh tranh càng cao thì khả năng áp dụng đổi mới công nghệ càng cao

Chứng nhận chất luọng: Soto-Acosta, Popa, và Martinez-Conesa (2018) nghiên cứu mô hình của đổi mới tại công DNVVN tại Tây Ban Nha. Kết quả cho thấy quản trị trí thức có ảnh hưởng quan trọng đến đổi mới. Trong đó việc thiết lập một hệ thống vận hành, các thủ tục, chuẩn hóa quy trình sẽ ảnh hướng tích cực đến việc đổi mới. Hệ thống này tương đồng với việc một doanh nghiệp có chứng nhận hệ thống chất lượng. Một doanh nghiệp có chứng nhận hệ thống chất lượng sẽ tạo ra một cơ chế quản lý tốt, từ đó thúc đẩy việc đổi mới. Chính vì vậy trong nghiên cứu này, nhóm tác giả đề xuất chứng nhận chất lượng là một biến độc lập có ảnh hướng đến đổi mới công nghệ trong tổ chức.

Từ đó nhóm tác giả đưa ra giả thuyết:

H4: Doanh nghiệp có khả năng có chứng nhận chất lượng càng cao thì khả năng áp dụng đổi mới công nghệ càng cao

Chính phủ: Theo Guo, Xia, Zhang, và Zhang (2018), sự hỗ trợ của chính phủ thông qua quỹ nghiên cứu và phát triển ảnh hưởng tích cực đến đổi mới tại các doanh nghiệp Trung Quốc. Jugend và cộng sự (2018) nghiên cứu tại công ty Brazil cũng kết luận sự hỗ trợ của chính phụ rất quan trọng trong việc áp dụng đổi mới. Không chỉ hỗ trợ về mặt tài chính, việc hỗ trợ về khía cạnh phi tài chính cũng rất quan trọng. Theo Liang và Liu (2018), sự hỗ trợ của chính phủ Trung Quốc trong việc thiết lập các mạng lưới hợp tác giữa các công ty đã giúp các doanh nghiệp quang điện mặt trời đổi mới công nghệ tốt hơn. Từ đó, nghiên cứu này đã đưa các yếu tố liên quan đến chính phủ làm biến độc lập với các biến quan sát là: sự hỗ trợ kỹ thuật của chính phủ, số lần kiểm tra về khía cạnh kỹ thuật của các cơ chức năng, và việc có phải chi trả các chi phí phi chính thức cho các lần kiểm tra hay không.

Từ đó nhóm tác giả đưa ra giả thuyết:

H5: Chính phủ có ảnh hưởng đến khả năng áp dụng đổi mới công nghệ, cụ thể: có hố trợ kỹ thuật của chính phủ càng cao thì khả năng áp dụng đổi mới công nghệ càng cao, số lần kiểm 
tra về khía cạnh kỹ thuật của các cơ chức năng càng cao thì khả năng áp dụng đổi mới công nghệ càng cao, việc chi trả các chi phí phi chính thức càng thấp thì khả năng áp dụng đổi mới công nghệ càng cao

\subsection{Mô hình nghiên cúu}

Từ những nghiên cứu thực nghiệm trên ta thấy có nhiều yếu tố tác động đến việc áp dụng các tiêu chuẩn chất lượng tại các doanh nghiệp. Để xác định được những yếu tố nào có tác động đến việc đổi mới công nghệ tại các DNVVN của Việt Nam, tác giả đề xuất mô hình nghiên cứu gồm các yếu tố đã được lược khảo ở các nghiên cứu trước như sau: Mức độ cạnh tranh, Đặc điểm chủ sở hũu, Quy mô doanh nghiệp, Chứng nhận chất lượng và Chính phủ. Bên cạnh đó, sau khi phỏng vấn chuyên sâu một số chuyên gia, nghiên cứu đề xuất thêm yếu tố Xuất khẩu và Đổi mới sản phẩm, theo các chuyên gia tại các Việt Nam hiện nay, các doanh nghiệp xuất khẩu thường có xu hướng đổi mới công nghệ nhiều hơn để theo kịp với môi trường bên ngoài; và việc đổi mới công nghệ thường theo sau việc đổi mới một sản phẩm nào đó, nhằm đảm bảo đổi mới sản phẩm thành công. Vì vậy tác giả đề xuất hai yếu tố Xuất khẩu và Đổi móti sản phẩm đóng vai trò làm hai biến giải thích của mô hình. Vậy mô hình bổ sung thêm hai giả thuyết nghiên cứu sau:

H6: Doanh nghiệp có khả năng xuất khẩu càng cao thì khả năng áp dụng đổi mới công nghệ càng cao

H7: Doanh nghiệp có khả năng đổi mới sản phẩm càng cao thì khả năng áp dụng đổi mới công nghệ càng cao

Cuối cùng, mô hình nghiên cứu được đề xuất như sau:

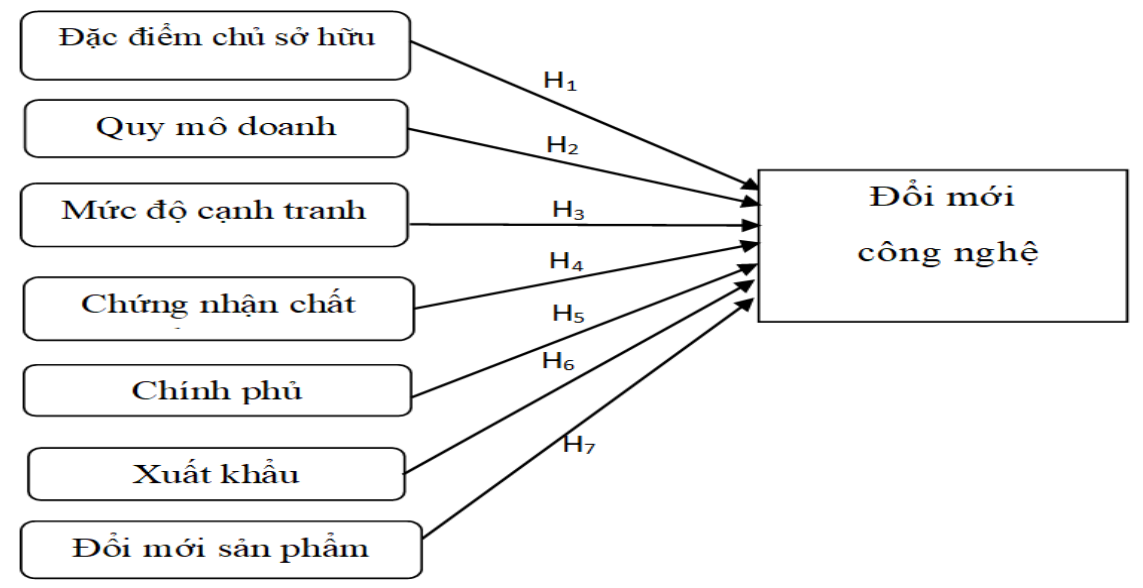

Hình 1. Mô hình nghiên cứu

Nguồn: Tổng hợp của tác giả

\section{Phương pháp nghiên cứu và dữ liệu}

\subsection{Phương pháp nghiên cúu}

Do biến phụ thuộc là biến định tính có hai trạng thái bao gồm có đổi mới công nghệ và không có đổi mới công nghệ nên ta có thể sử dụng mô hình hồi quy Logit hoặc Probit để phân tích dữ liệu. Các nghiên cứu thực nghiên cho thấy không có sự khác biệt lớn về kết quả hồi quy giữa mô hình Logit và Probit (Gujarati, 2004). Do đó, trong nghiên cứu này, tác giả sử dụng phương pháp hồi quy logit nhằm xác định những yếu tố có ảnh hưởng đến việc đổi mới công nghệ tại các DNVVN.

Theo Gujarati (2004), mô hình hồi quy logit được trình bày như sau: 


$$
\ln \left(\frac{P_{i}}{1-P_{i}}\right)=\beta_{1}+\beta_{2} X_{i}+u_{i}
$$

Trong đó $\mathrm{P}_{\mathrm{i}}=\operatorname{Pr}\left(\mathrm{Y}_{\mathrm{i}}=1 / \mathrm{X}_{\mathrm{i}}\right)$ là xác suất để $\mathrm{Y}_{\mathrm{i}}$ nhận giá trị 1 với giá trị $\mathrm{X}_{\mathrm{i}}$ cho trước hay là xác suất để doanh nghiệp có đổi mới công nghệ với các đặc điểm cho trước của doanh nghiệp. $\mathrm{X}_{\mathrm{i}}$ là vector các biến độc lập, $\beta_{1}$ là hằng số và $\beta_{2}$ là vectơ các hệ số hồi quy, $\mathrm{u}_{\mathrm{i}}$ là sai số. Mô tả chi tiết cho việc mã hóa các biến số được trình bày trong bảng sau đây.

\section{Bảng 1}

Giải thích các biến sử dụng trong mô hình

\begin{tabular}{|c|c|}
\hline Tên biến & Giải thích/đại diện biến \\
\hline Đổi mới công nghệ & $\begin{array}{l}\text { 1: Doanh nghiệp có đổi mới công nghệ, quy trình } \\
\text { 0: Doanh nghiệp không đổi mới công nghệ, quy trình }\end{array}$ \\
\hline Chi phí R\&D & Chi phí đầu tư nghiên cứu và phát triển, đơn vị tính: triệu VNĐ \\
\hline $\begin{array}{l}\text { Qy mô doanh nghiệp } \\
\text { (Doanh thu) }\end{array}$ & $\begin{array}{l}\text { Đại diện bởi tổng doanh thu của doanh nghiệp năm 2014, đơn vị tính: triệu } \\
\text { VNĐ }\end{array}$ \\
\hline Ý tưởng đổi mới & $\begin{array}{l}\text { Ý tưởng đổi mới của chủ doanh nghiệp hoặc người quản lý, mức độ từ 1-5: } \\
\text { 1: Không có ý tưởng đổi mới } \\
\text { 5: Có rất nhiều ý tưởng đổi mới }\end{array}$ \\
\hline Né tránh rủi ro & $\begin{array}{l}\text { Thái độ né tránh rủi ro của chủ doanh nghiệp hoặc người quản lý, mức độ từ } \\
0-10 \text { : } \\
\text { 0: Rất sợ rủi ro } \\
\text { 10: Rất thích rủi ro }\end{array}$ \\
\hline Số lần kiểm tra & $\begin{array}{l}\text { Số lần các cơ quan chức năng đi kiểm tra liên quan đến kỹ thuật trong năm } \\
2014\end{array}$ \\
\hline Số lượng lao động & Tổng số lao động trong công ty tính đến cuối năm 2014 \\
\hline Giới tính chủ sở hữu & $\begin{array}{l}\text { 1: Nam } \\
0: \text { Nũ }\end{array}$ \\
\hline $\begin{array}{l}\text { Trình độ học vấn chủ sở } \\
\text { hữu }\end{array}$ & $\begin{array}{l}\text { 1: Có trình độ trung cấp nghề trở lên } \\
\text { 0: Không có trình độ trung cấp nghề trở xuống }\end{array}$ \\
\hline Cạnh tranh & $\begin{array}{l}\text { 1: Có cạnh tranh } \\
\text { 0: Không có cạnh tranh }\end{array}$ \\
\hline Loại hình doanh nghiệp & $\begin{array}{l}\text { 1: Doanh nghiệp là doanh nghiệp hộ gia đình } \\
\text { 0: Các loại hình doanh nghiệp khác }\end{array}$ \\
\hline $\begin{array}{l}\text { Chứng nhận chất lượng } \\
\text { quốc tế }\end{array}$ & $\begin{array}{l}\text { 1: Doanh nghiệp có đạt giấy chứng nhận chất lượng quốc tế } \\
\text { 0: Doanh nghiệp không đạt giấy chứng nhận chất lượng quốc tế }\end{array}$ \\
\hline $\begin{array}{l}\text { Chứng nhận chất lượng } \\
\text { trong nước }\end{array}$ & $\begin{array}{l}\text { 1: Doanh nghiệp có đạt giấy chứng nhận chất lượng trong nước } \\
\text { 0: Doanh nghiệp không đạt giấy chứng nhận chất lượng trong nước }\end{array}$ \\
\hline Xuất khẩu & $\begin{array}{l}\text { 1: Doanh nghiệp có xuất khẩu } \\
\text { 0: Doanh nghiệp không xuất khẩu }\end{array}$ \\
\hline Chi phí phi chính thức & 1: Doanh nghiệp có phải chi trả chi phí phi chính thúc \\
\hline
\end{tabular}




\begin{tabular}{|l|l|}
\hline \multicolumn{1}{|c|}{ Tên biến } & \multicolumn{1}{c|}{ Giải thích/dại diện biến } \\
\hline & 0: Doanh nghiệp không phải chi trả chi phí phi chính thức \\
\hline Đổi mới sản phẩm & $\begin{array}{l}\text { 1: Doanh nghiệp có đổi mới sản phẩm/ dịch vụ } \\
\text { 0: Doanh nghiệp không đổi mới sản phâm/ dịch vụ }\end{array}$ \\
\hline \multirow{3}{*}{ Sự hỗ trợ của chính phủ } & $\begin{array}{l}\text { Chính phủ có hỗ trợ kỹ thuật (technical assistance) trong năm 2014 cho } \\
\text { doanh nghiệp không: } \\
\text { 1: Có hỗ trợ } \\
\text { 0: Không hỗ trợ }\end{array}$ \\
\hline
\end{tabular}

Nguồn: Tổng hợp của tác giả

\subsection{Dũ liệu nghiên cúu}

Đề tài tiếp cận dựa vào bộ dữ liệu khảo sát DNVVN năm $2015^{1}$ được thu thập bởi Viện khoa học lao động và xã hội (ILSSA) thuộc Bộ Lao động Thương binh và Xã hội (MOLISA), Viện nghiên cứu quản lý kinh tế trung ương (CIEM) thuộc Bộ Kế hoạch \& đầu tư (MPI), và Khoa kinh tế (DoE) thuộc Đại học Copenhagen cùng Đại sứ quán Đan Mạch tại Việt Nam. Năm 2015 là lần khảo sát thứ 6 (điều tra 2 năm một lần). Trong cuộc khảo sát năm 2015 có 2649 DNNVV ngoài quốc doanh hoạt động trong lĩnh vực chế biến, chế tạo tại trên lãnh thổ Việt Nam. Đối tượng trả lời phỏng vấn là chủ sở hữu doanh nghiệp hoặc là nhà quản lý.

\section{Kết quả nghiên cứu}

\subsection{Thống kê mô tả}

Dựa vào kết quả thống kê mô tả đối với biến phụ thuộc ta thấy, trong 2649 DNNVV được khảo sát thì có 2520 doanh nghiệp không đổi mới công nghệ (chiếm 95,1\%), chỉ có 129 DNNVV đổi mới công nghệ (chiếm 4,9\%).

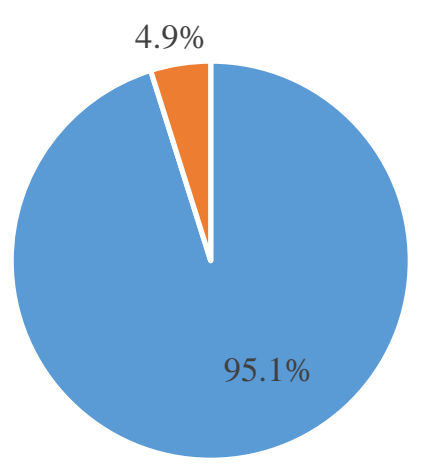

- Không đổi mới công nghệ Có đổi mới công nghệ

Hình 2. Tỷ lệ DNVVN đổi mới công nghệ

Tiếp theo, Hình 3 thể hiện mối liên hệ việc chính phủ có hỗ trợ kỹ thuật và đổi mới công nghệ của doanh nghiệp. Sự hỗ trợ của chính phủ càng cao thì tỷ lệ đạt doanh nghiệp đổi mới công nghệ càng cao. Cụ thể, nếu chính phủ có hỗ trợ thì tỷ lệ đạt đổi mới công nghệ là $29,4 \%$. Ngược lại tỷ lệ sẽ là 4,7\% nếu chính phủ không hỗ trợ.

\footnotetext{
${ }^{1}$ Hiện tại bộ dữ liệu năm 2015 vẫn đang là bộ dữ liệu khảo sát DNVVN mới nhất được công bố.
} 


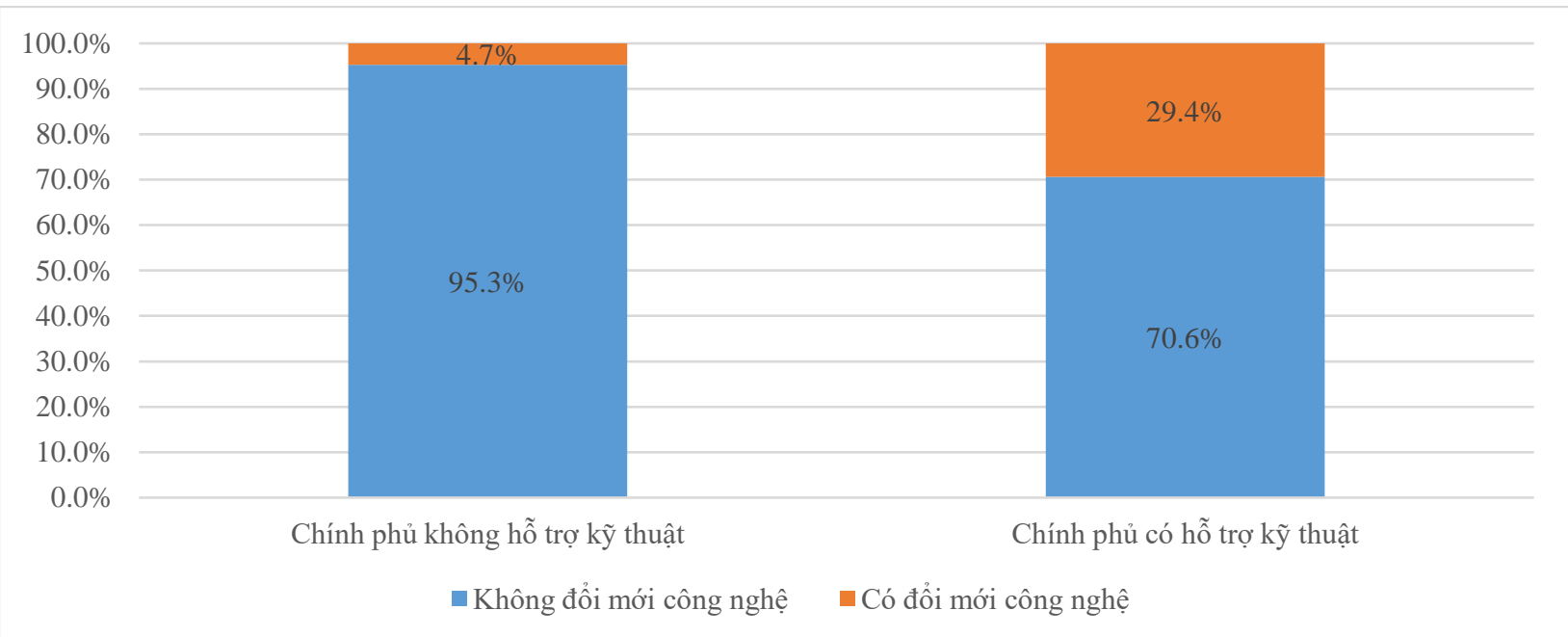

Hình 3. Mối quan hệ sự hỗ trợ của chính phủ và khả năng đổi mới công nghệ

\subsection{Kết quả hồi quy logit}

Để thấy rõ hơn mối liên hệ giữa biến phụ thuộc và các biến độc lập nhóm tác giả đã đi thực hiện hồi quy logit với biến phụ thuộc là khả năng đổi mới công nghệ. Chi tiết kết quả về mô hình hồi quy được trình bày sau đây.

\section{Bảng 2}

Kết quả hồi quy mô hình

\begin{tabular}{|l|r|r|r|r|r|}
\hline \multicolumn{1}{|c|}{ Biến số } & \multicolumn{1}{c|}{ Hệ số } & Sai số chuẩn & Giá trị T & Giá trị P & Hệ số VIF \\
\hline Hằng số & -0.22710 & 0.0390 & -5.83 & 0.000 & \\
\hline Chi phí nghiên cứu và phát triển & 0.00000 & 0.0000 & 0.69 & 0.490 & 1.01 \\
\hline Quy mô doanh nghiệp (Doanh thu) & $0.04343 * * *$ & 0.0059 & 7.24 & 0.000 & 1.20 \\
\hline Ý tưởng đổi mới & 0.00006 & 0.0036 & 0.02 & 0.986 & 1.07 \\
\hline Né tránh rủi ro & -0.00126 & 0.0017 & -0.73 & 0.468 & 1.05 \\
\hline Số lần kiềm tra & $0.01463 * *$ & 0.0065 & 2.26 & 0.024 & 1.19 \\
\hline Số lượng lao động & -0.00016 & 0.0001 & -1.22 & 0.224 & 1.42 \\
\hline Giới tính & -0.00397 & 0.0084 & -0.47 & 0.638 & 1.05 \\
\hline Trình độ học vấn chủ sơ hữu & -0.00690 & 0.0121 & -0.57 & 0.566 & 1.42 \\
\hline Cạnh tranh & 0.00100 & 0.0126 & 0.08 & 0.935 & 1.05 \\
\hline Loại hình doanh nghiệp & 0.00270 & 0.0112 & 0.24 & 0.807 & 1.79 \\
\hline Chứng nhận chất lượng quốc tế & $0.04260 *$ & 0.0229 & 1.86 & 0.063 & 1.18 \\
\hline Chứng nhận chất lượng trong nước & -0.01870 & 0.0125 & -1.50 & 0.134 & 1.07 \\
\hline Xuất khẩu & $0.03000 *$ & 0.0178 & 1.68 & 0.093 & 1.27 \\
\hline Chi phí phi chính thức & $-0.01810^{*}$ & 0.0093 & -1.94 & 0.053 & 1.31 \\
\hline Đổi mới sản phẩm & $0.08630 * * *$ & 0.0101 & 8.52 & 0.000 & 1.08 \\
\hline Hỗ trợ kỹ thuật & $0.19470 * * *$ & 0.0509 & 3.83 & 0.000 & 1.01 \\
\hline
\end{tabular}

Ghi chú: ký hiệu*, ** và *** đại diện cho mức ý nghĩa thống kê $10 \%, 5 \%$ và $1 \%$.

Nguồn: Phân tích của tác giả 
Các tác giả tiến hành dò tìm các vi phạm giả định hồi quy theo đúng kỹ thuật phân tích Logit. Cụ thể, nhóm đã kiểm định phương sai sai số thay đổi và tính chất đa cộng tuyến của mô hình. Kết quả kiểm định White cho thấy mức ý nghĩa của kiểm định $\operatorname{Prob}(\mathrm{F})>10 \%$ nên nhóm kết luận không xảy ra hiện tượng phương sai sai số thay đổi. Kết quả kiểm định đa cộng tuyến được thể hiện ở hệ số VIF ở Bảng 2. Tất cả các hệ số phóng đại VIF đều bé hơn hai nên ta có thể kết luận phương trình hồi quy không có hiện tượng đa cộng tuyến.

Cuối cùng, nhóm kết luận với biến phụ thuộc là đổi mới công nghệ, các biến độc lập có ảnh hưởng bao gồm: quy mô doanh nghiệp, số lần kiểm tra, chứng nhận chất lượng quốc tế, xuất khẩu, chi phí phi chính thức, đổi mới sản phẩm, hỗ trợ kỹ thuật.

\subsection{Thảo luận kết quả nghiên cúu}

Từ kết quả phân tích ta thấy, quy mô doanh nghiệp (doanh thu), đổi mới sản phẩm và hỗ trợ kỹ thuật là các biến số có ảnh hưởng lớn nhất tới việc đổi mới công nghệ với mức ý nghĩa thống kê < $1 \%$ trong mô hình. Kết quả này cũng tương đồng với kết quả của nhiều nghiên cứu trước. Nếu doanh nghiệp có doanh thu càng cao thì khả năng đổi mới công nghệ càng cao, điều này đã được các nhà nghiên cứu trước giải thích là các công ty lớn thường có nguồn lực về tài chính, khả năng nghiên cứu mạnh mẽ hơn, và hệ thống vận hành tốt hơn nên dễ dàng trong việc đổi mới công nghệ hơn các công ty nhỏ. Bên cạnh đó, nếu doanh nghiệp có ý định đổi mới sản phẩm thì họ thường đổi mới công nghệ phù hợp với sản phẩm chuẩn bị sản xuất. Đó là lý do tại sao đổi mới sản phẩm là một biến có ảnh hưởng lớn đến đổi mới công nghệ.

Việc hỗ trợ kỹ thuật của chính phủ mang ý nghĩa quan trọng trong việc đổi mới công nghệ tại các DNVVN tại Việt Nam. Nếu chính phủ có sự hỗ trợ đối với doanh nghiệp thì DNVVN sẽ dễ dàng tiếp cận các công nghệ mới, thay đổi các công nghệ lạc hậu để có thể sản xuất ra các sản phẩm chất lượng đáp ứng nhu cầu khách hàng.

Biến số tiếp theo ảnh hưởng đến khả năng đổi mới công nghệ là số lần kiểm tra về kỹ thuật của cơ quan chức năng và việc có phải chi trả chi phí phi chính thức không. Nếu số lần kiểm tra liên quan đến kỹ thuật, công nghệ của cơ quan càng nhiều thì khả năng doanh nghiệp đổi mới công nghệ càng cao, ngược lại nếu doanh nghiệp phải trả chi phí phi chính thức thì khả năng đổi mới công nghệ lại càng thấp. Kết quả này cho thấy, các chính sách kiểm tra giám sát của các cơ quan chức năng có ý nghĩa tích cực, nhằm giúp doanh nghiệp đổi mới công nghệ, tìm phương án sản xuất hiệu quả, đảm bảo môi trường làm việc cũng như chất lượng sản phẩm. Tuy nhiên các chính sách này sẽ thực sự hiệu quả nếu việc kiểm tra minh bạch, công tâm và chính xác. Việc có chi trả chi phí không chính thức là rào cản để doanh nghiệp cải tiến, nâng cao chất lượng sản phẩm cũng như đổi mới công nghệ.

Cuối cùng, biến chứng nhận chất lượng quốc tế và xuất khẩu có ảnh hưởng lớn đến việc đổi mới công nghệ với mức ý nghĩa $10 \%$. Dường như các yếu tố nước ngoài có ảnh hưởng tích cực đến đối mới công nghệ. Các doanh nghiệp xuất khẩu và doanh nghiệp có giấy chứng nhận quốc tế đều có xu hướng đổi mới công nghệ tốt hơn các doanh nghiệp còn lại.

\section{Hàm ý chính sách}

Từ kết quả nghiên cứu ta thấy, có bảy nhân tố có ảnh hưởng đến đổi mới công nghệ tại các DNVVN. Do đó, để nâng cao khả năng đổi mới công nghệ của các doanh nghiệp các nhà hoạch định chính sách có thể áp dụng các giải pháp sau.

Thứ nhất, hỗ trợ của chính phủ có ảnh hưởng rất lớn đến việc doanh nghiệp có đổi mới công nghệ hay không, chính vì vậy chính phủ cần có nhiều chính sách hỗ trợ DNNVV đổi mới công nghệ dựa trên ứng dụng và chuyển giao công nghệ, làm chủ các công nghệ then chốt, nâng 
cao năng suất, chất lượng và sức cạnh tranh của sản phẩm, áp dụng hệ thống quản lý chất lượng tiên tiến, công cụ cải tiến năng suất, chất lượng thích hợp với DNNVV. Chính phủ có thể hỗ trợ tạo các mạng lưới liên kết chuỗi để các DNVVN có thể chia sẻ công nghệ kỹ thuật với nhau, thúc đẩy nhau đổi mới công nghệ, nâng cao năng suất và chất lượng.

Do nguồn lực có hạn nên trước hết Việt Nam có thể tập trung hỗ trợ một vài lĩnh vực trọng điểm. Ví dụ như lĩnh vực dịch vụ ăn uống, nhà hàng khách sạn và các dịch vụ du lịch. Số lượng các DNVVN hoạt động trong lĩnh vực này khá nhiều, và nhu cầu khách hàng trong lĩnh vực này ngày càng tăng. Việc đổi mới công nghệ sẽ mang lại lợi thế cạnh tranh vô cùng to lớn trong lĩnh vực đầy tiềm năng này.

Thư hai, bên cạnh việc hỗ trợ thì việc thanh tra, giám sát cũng rất quan trọng. Có nhiều chuyên gia cho rằng Việt Nam đang dẫn trở thành bãi rác công nghệ của thế giới. Những công nghệ lạc hậu, gây ô nhiễm môi trường khi các chính phủ nước ngoài cấm sử dụng, đã được nhập về Việt Nam. Việc nhập khẩu máy móc, công nghệ lạc hậu, phế liệu rác thải không chỉ trực tiếp đe dọa môi trường, đe dọa an ninh năng lượng, mà còn cản trở quá trình hiện đại hóa nền công nghiệp của đất nước, giảm giá trị cạnh tranh của quốc gia. Theo đánh giá của ông Phan Xuân Dũng (Chủ nhiệm Ủy ban Khoa học, Công nghệ và Môi trường của Quốc hội) : “Tốc độ đổi mới công nghệ trong các doanh nghiệp thời gian qua rất thấp, chưa đạt như mong muốn (10\%/năm). Một số ngành, lĩnh vực như nhiệt điện, xi-măng, mía đường, luyện cán thép và khai khoáng vẫn sử dụng công nghệ lạc hậu. Chúng ta vẫn chuyển giao công nghệ thông qua mua máy móc, thiết bị phần lớn đã lạc hậu hai, ba thế hệ là chính. Điều này ảnh hưởng trực tiếp đến hiệu quả và sức cạnh tranh của nền kinh tế nước ta”. Còn theo ông Chu Ngọc Anh (Bộ trưởng Khoa học Công nghệ) đánh giá tình trạng công nghệ trong nước là "rất yếu kém". Điều này thể hiện qua các số liệu: năm 2015 , Việt Nam đứng thứ $56 / 140$ trong bảng xếp hạng cạnh tranh toàn cầu, nhưng mức độ sẵn sàng về công nghệ chỉ đứng thứ 92, mức độ hấp thụ công nghệ của doanh nghiệp đứng thứ 121 và khả năng tiếp thụ công nghệ đứng thứ 112 trong số 140 quốc gia được đánh giá. Đáng lo ngại hơn, theo Bộ trưởng Chu Ngọc Anh, là chúng ta vẫn thiếu công cụ pháp lý để kiểm soát và ngăn chặn các luồng công nghệ lạc hậu được nhập vào trong nước (Song Giang, 2016). Chính vì vậy việc hoàn thiện bộ khung pháp lý cũng như các quy định chặt chẽ trong việc thanh tra giám sát đổi mới công nghệ là vấn đề mà các nhà hoạch định cần quan tâm.

Cuối cùng, các doanh nghiệp có chứng nhận chất lượng quốc tế thì khả năng đổi mới công nghệ càng cao, nên việc khuyến khích các DNVVN áp dụng các chứng nhận chất lượng quốc tế là một giải pháp bền vững. doanh nghiệp sẽ có một hệ thống quản lý tốt, vận hành hiệu quả giúp doanh nghiệp dễ dàng tiếp cận đổi mới công nghệ thành công hơn. Hiện nay có rất nhiều phương pháp quản lý trên thế giới mà các doanh nghiệp Việt Nam có thể áp dụng như: ISO 9001, ISO 14001, ISO 22000 và ISO 27000. Tùy vào từng ngành nghề, lĩnh vực hoạt động khác nhau mà các doanh nghiệp tự lựa chọn cho mình mô hình quản lý phù hợp nhất.

Hạn chế đề tài: Mặc dù nghiên cứu đã đạt được một số kết quả, tuy nhiên nghiên cứu còn tồn tại một số hạn chế sau. Đề tài mới dừng lại ở việc phân tích dữ liệu thời điểm, chưa phân tích được ở dạng dữ liệu bảng để thấy được sự biến động của biến phụ thuộc theo thời gian. Đề tài chỉ mới kiểm chứng kết quả bằng mô hình hồi quy logit mà chưa so sánh được kết quả với các mô hình khác. Tác giả hy vọng sẽ khắc phục các hạn chế trên trong các nghiên cứu tiếp theo. 


\section{Tài liệu tham khảo}

Azadegan, A., Patel, P. C., \& Parida, V. (2013). Operational slack and venture survival. Production and Operations Management, 22(1), 1-18.

Bhasin, K. (2012). This is the difference between "invention" and "innovation". Retrieved July 01, 2018, from Business insider website: https://www.businessinsider.com/this-is-thedifference-between-invention-and-innovation-2012-4

Branscomb, L. M. (2001). Technological innovation. International Encyclopedia of the Social \& Behavioral Sciences, 2001(5), 498-502.

Branzei, O., \& Vertinsky, I. (2006). Strategic pathways to product innovation capabilities in SMEs. Journal of Business Venturing, 21(1), 75-105.

Cancino, C. A., Paza, A. I. L., Ramaprasad, A., \& Syn, T. (2018). Technological innovation for sustainable growth: An ontological perspective. Journal of Cleaner Production, 179, 31-41.

Divisekera, S., \& Nguyen, K. V. (2018). Determinants of innovation in tourism evidence from Australia. Tourism Management, 67, 157-167.

Frankelius, P. (2009). Questioning two myths in innovation literature. The Journal of High Technology Management Research, 20(1), 40-51.

Gnyawali, D. R., \& Park, B.-J. (2009). Co-opetition and technological innovation in small and medium-sized enterprises: A multilevel conceptual model. Journal of Small Business Management, 47(3), 308-330.

Gujarati, D. N. (2004). Basic econometrics. New York, NY: McGraw-Hill.

Guo, Y., Xia, X., Zhang, S., \& Zhang, D. (2018). Environmental regulation, government R\&D funding and green technology innovation: Evidence from China provincial data. Sustainability, 10(4), 940.

Jugend, D., Jabbour, C. J. C., Scaliza, J. A. A., Rocha, R. S., Junior, J. A. G., ... Salgado, M. H. (2018). Relationships among open innovation, innovative performance, government support and firm size: Comparing Brazilian firms embracing different levels of radicalism in innovation. Technovation, 74-75, 54-65.

Kim Hien (2015). Đổi mới công nghệ để doanh nghiệp "cất cánh” [Innovating technology for businesses to "take off"']. Retrieved February 25, 2019, from http://kinhtevadubao.vn/chitiet/146-4372-doi-moicong-nghe-de-doanh-nghiep-cat-canh.html, 2015.

Liang, X., \& Liu, A. M. M. (2018). The evolution of government sponsored collaboration network and its impact on innovation: A bibliometric analysis in the Chinese solar PV sector. Research Policy, 47(7), 1295-1308.

Lubatkin, M. H., Simsek, Z., Ling, Y., \& Veiga, J. F. (2006). Ambidexterity and performance in small-to medium-sized firms: The pivotal role of top management team behavioral integration. Journal of Management, 1, 32-64.

Minh Nhat (2015). Bài toán đổi mới công nghệ cho doanh nghiệp [Technology innovation problem for businesses]. Retrieved February 27, 2019, from http://www.nhandan.com.vn/kinhte/tintuc/item/26912402-bai-toan-doi-moi-cong-nghe-cho-doanh-nghiep.html

Nguyen, T. A., Vu, H. T., \& Nguyen, M. T. T. (2018). Hỗ trợ doanh nghiệp nhỏ và vừa tiếp cận các quỹ đổi mới công nghệ nhà nước: Kinh nghiệm quốc tế và bài học cho Việt Nam 
[Supporting small and medium enterprises to access state technology innovation funds: International experiences and lessons for Vietnam]. Tạp chí Khoa hoc ĐHQGHN: Kinh tế và Kinh doanh, 34(1), 15-23.

OECD. (2005). Growth in services. Paper presented at the Meeting of the OECD Council at Ministerial Level, 2005.

Raisch, S., \& Birkinshaw, J. (2008). Organizational ambidexterity: Antecedents, outcomes, and moderators. Journal of Management, 34(3), 375-409.

Rangus, K., \& Slavec, A. (2017). The interplay of decentralization, employee involvement and absorptive capacity on firms' innovation and business performance. Technological Forecasting \& Social Change, 120(C), 195-203.

Sarooghi, H., Libaers, D., \& Burkemper, A. (2015). Examining the relationship between creativity and innovation: A meta-analysis of organizational, cultural, and environmental factors. Journal of Business Venturing, 30(5), 714-731.

Song Giang. (2016). "Bãi rác công nghệ" - không còn là cảnh báo ["Technology landfill" - no longer a warning]. Retrieved September 20, 2019, from https://www.nhandan.com.vn/ chinhtri/item/30706102-\%E2\%80\%9Cbai-rac-cong-nghe\%E2\%80\%9D-khong-con-lacanh-bao.html

Soto-Acosta, P., Popa, S., \& Martinez-Conesa, I. (2018). Information technology, knowledge management and environmental dynamism as drivers of innovation ambidexterity: A study in SMEs. Journal of Knowledge Management, 22(4), 824-849.

Souitaris, V. (2002). Technological trajectories as moderators of firm-level determinants of innovation. Research Policy, 31(6), 877-898.

Thong, J. Y. L. (2015). An integrated model of information systems adoption in small businesses. Journal of Management Information Systems, 15(4), 187-214.

UNITED NATIONS UNIVERSITY. (2015). Characteristics of the Vietnamese business environment: Evidence from a SME survey in 2015. Retrieved September 20, 2019, from https://www.wider.unu.edu/publication/characteristics-vietnamese-business-environment

UNITED NATIONS UNIVERSITY. (2015). Viet Nam SMEs database. Retrieved October 25, 2018, from https://www.wider.unu.edu/database/viet-nam-sme-database

Uzkurt, C., Kumar, R., Kimzan, H. S., \& Sert, H. (2012). The impact of environmental uncertainty dimension on organizational innovavtiveness: An empirical study on SMEs. International Journal of Innovation Management, 16(2), 1-23. 